\title{
A Centrifugo-Magnetically ACtuated Gas Micropump
}

\author{
S. Haeberle ${ }^{1}$, N. Schmitt ${ }^{1}$, R. Zengerle ${ }^{1,2}, J$. Ducrè $^{2}$ \\ ${ }^{1}$ University of Freiburg - IMTEK, Laboratory for MEMS Applications, \\ Georges-Koehler-Allee 106, D-79110 Freiburg, Germany \\ ${ }^{2}$ HSG-IMIT, Wilhelm-Schickard Strasse 10, D-78052 Villingen-Schwenningen, Germany \\ e-mail: haeberle@imtek.de
}

\begin{abstract}
This paper describes a novel gas micropump on a centrifugal microfluidic platform. The pump is integrated on a passive and microstructured polymer disk which is sealed with an elastomer lid featuring paramagnetic inlays. The rotational motion of this hybrid disk over a stationary magnet induces a designated sequence of volume displacements of the elastic lid, leading to a net transport of gas. The pumping pressure scales linearly with the frequency, with a maximum observable pressure of $4.1 \mathrm{kPa}$.

The first application of this rotary device is the production of gas-liquid flows by pumping ambient air into a continuous centrifugal flow of liquid. The injected gas volume segments the liquid stream into a series of liquid compartments. Apart from such multi-phase flows, the new pumping technique supplements a generic air-to-liquid sampling method to centrifugal microfluidic platforms.
\end{abstract}

\section{INTRODUCTION}

The toolbox of centrifugal microfluidics has been continuously extended within the last years. Up to now, applications in (bio-)analytics [1,2] as well as a diverse set of unit operations such as cell-lysis [3], continuous micromixing [4], hematocrit determination [5] and emulsification [6] have been successfully implemented. However, the processing of gases in rotating micro-channels still remains a challenge since the centrifugal pumping force scales with the fluid density, thus reducing the force density on gases by three orders of magnitude with respect to liquids. In particular the generation of gas-liquid flows is aggravated by the buoyancy of the gas under the strong "artificial-gravity" conditions of the centrifugal field.

To access the interesting field of gas-liquid flows [7] with our centrifugal platform, the gas has to be differentially pressurized. To comply with the modular concept of the rotary system, the typically disposable disk should remain passive and the force transmission ought to be accomplished in a contact-free fashion. We chose a centrifugo-magnetical principle to displace movable, stationary disk-based steel plates by a permanent magnet aligned along the orbit of the pumping chamber [8].

Several magnetically driven micro-pumps were presented recently where external electro-magnets deflect permanent magnets integrated on elastic membranes [9-11].
Silicone elastomers, predominantly PDMS, are commonly used due to their simple handling, adjustable elastic properties as well as their cost-efficiency $[12,13]$. Compared to these approaches, our novel design significantly simplifies the setup by drawing the power for both, the centrifugal liquid pumping as well as the pressurization of the gas, quasi-independently from a single, rotary power source.

This paper starts with an outline of the functional principle followed by the device fabrication and the experimental results.

\section{FUNCTIONAL PRINCIPLE}

Two chambers of our novel, centrifugo-magnetically actuated micropump are located at the same radial but different azimuthal positions. They are sealed by a flexible PDMS-lid incorporating metallic inlays (Fig. 1). The outlet of the pump is connected to a microfluidic channel network.

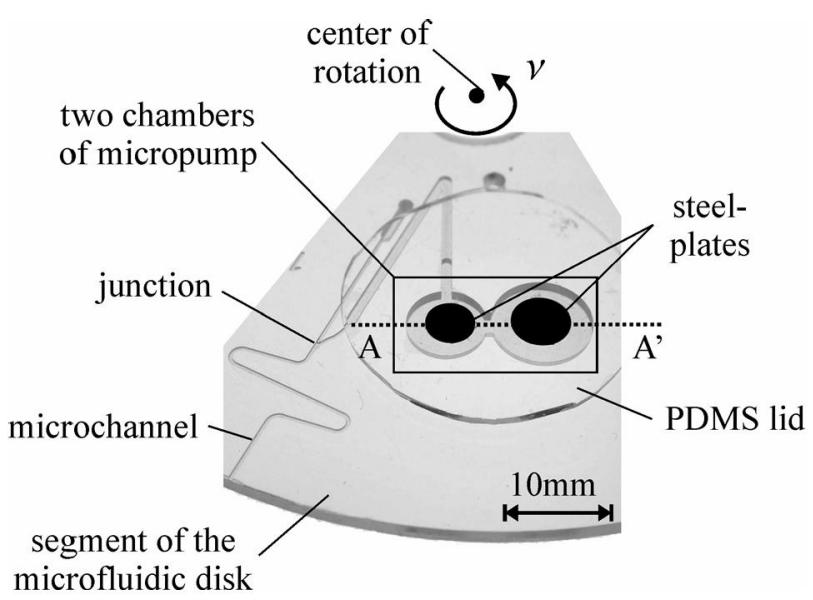

Figure 1: Sector of the microfluidic disk featuring a network of sealed microchannels. A thin PDMS lid with a thickness of roughly $0.7 \mathrm{~mm}$ and integrated steel plates is placed above the two pump chambers. The disk spins at a rotational frequency $v$. This way, liquid is pumped centrifugally through the microchannels, and, in addition, environmental gas is pumped by the sequential displacement of the membrane into the pump chambers while passing a stationary permant magnet (see Fig. 2). 


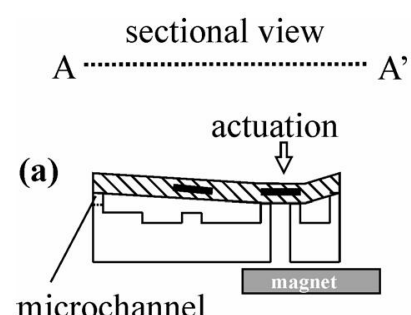

microchannel

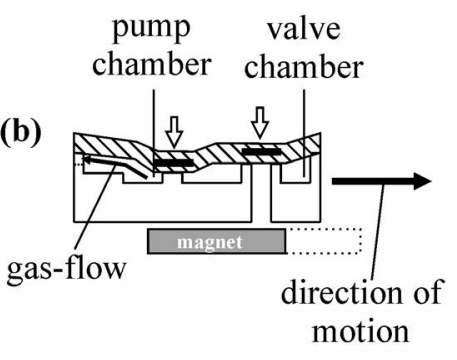

(c)

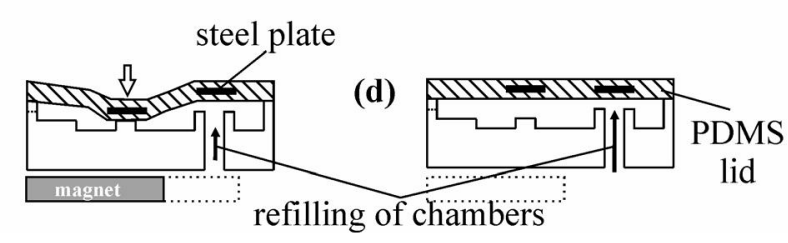

Figure 2: Functional principle of the gas micropump. The pump chamber orbits above a stationary mounted permanent magnet. The two steel-plates within the PDMSlid are spaced in azimuthal direction to induce a defined sequence of displacements during rotation for pressurizing the gas. After the magnet has passed, the chambers are refilled with ambient gas through the orifice of the valve chamber.

The steel plate inlays above the chambers pass a conventional permanent magnet placed at a fixed position along the orbit of the pump. The magnetic force triggers two phase-shifted displacements in the chambers (Fig. 2). The pumping sequence exhibits four stages: (a) closing of the valve with the PDMS-membrane at the seat of the valve chamber, (b) displacement of a defined gas volume out of the pump chamber into the connected microchannel (since the valve is now closed). Afterwards (c), the permanent magnet has passed the valve chamber, and the orifice is opened again. In the last stage (d), the pump chamber is refilled with ambient gas due to the relaxation of the membrane (the magnet has now also passed the pump chamber). Within the last step, the small hydrodynamic resistance of the valve orifice suppresses backflow from the microchannel into the actuation chamber.

The elastomer lid housing the metal inlays constitutes the only moving part of the passive disk while the macroscopic centrifuge periodically actuates the micropump each time the micropump passes the fixed permanent magnet. By arranging more than one magnet on the orbit of the rotating pump, several pumping cycles per revolution can be realized.

\section{FABRICATION}

The channels and orifices are micromachined into the polymer substrate by precision engineering processes (Fig. 3 ). The pump chamber measures a diameter of $8 \mathrm{~mm}$ and a depth of $1.2 \mathrm{~mm}$, featuring a bump with a height of $0.7 \mathrm{~mm}$ and a diameter of $2.5 \mathrm{~mm}$ at its center. The valve chamber possesses a diameter of $10 \mathrm{~mm}$ and a depth of $1.2 \mathrm{~mm}$, featuring an additional valve seat with a width of $0.3 \mathrm{~mm}$ and a distance of $0.2 \mathrm{~mm}$ to the membrane around the $2.6 \mathrm{~mm}$ diameter valve orifice.

The microfluidic channel network is (partially) hydrophobically coated and sealed by a transparent adhesive tape to observe the enclosed liquid under rotation at a frequency $v$ with a stroboscopic measurement setup [14]. The self-adhesive PDMS-lid is cast with two integrated steel plates (thickness: $400 \mu \mathrm{m}$, diameter: $5 \mathrm{~mm}$ and $6 \mathrm{~mm}$, respectively) and cured resulting in an approximately $0.7 \mathrm{~mm}$ thick elastic lid. The steel plates themselves are manufactured by wire electrical discharge machining (WEDM) from standard spring steel. The PDMS lid is aligned above the two chambers where the adhesive tape has been removed, so that the center of the disk shaped steel plates matches the bump of the pump and the orifice of the valve chamber, respectively. Afterwards, the lid is bonded to the disk-surface by adhesion.

\section{EXPERIMENTAL RESULTS}

For all the experiments, the disk is mounted up-side down on a centrifugal drive so that the magnet can be positioned on the pump orbit in a distance of approximately $2 \mathrm{~mm}$ to the disk.

The first series of experiments visualizes the maximum pressure head of the gas pump. To this end, the pump is connected to the left arm of the U-shaped channel (width: $1 \mathrm{~mm}$, depth: $500 \mu \mathrm{m}$ ) in Fig. 4. A plug of ink-colored DIwater is introduced into the channel through the right arm that remains open, thus acting as an air vent. Upon rotation, the two liquid-air interfaces of the water plug tend to level out by the principle of interconnected tubes in the strong centrifugal field of "artificial gravity".

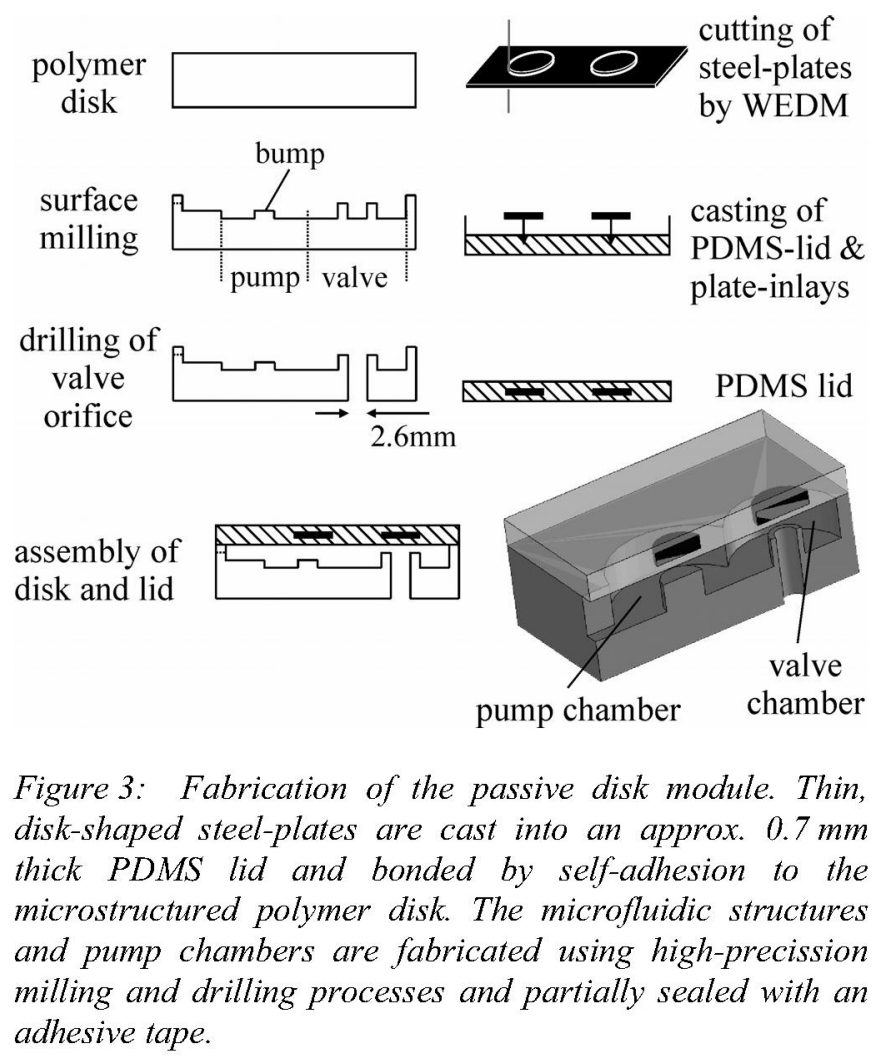



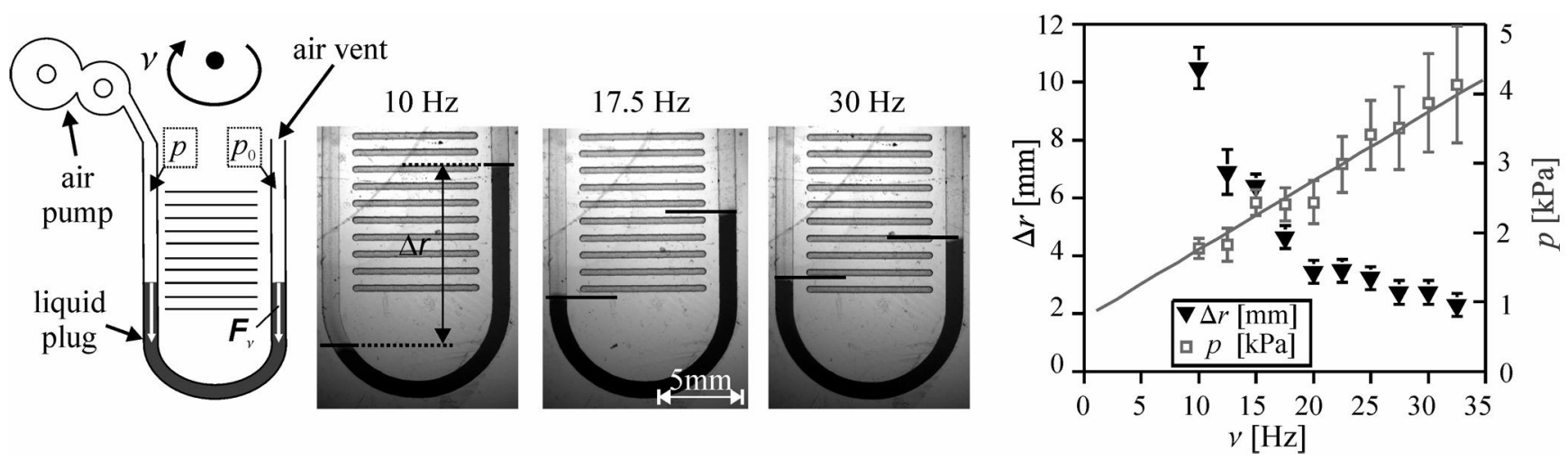

Figure 4: Visualization of the pressure head by a U-shaped channel (width: 1 mm, depth: 500 um). Depending on the frequency of rotation $v$, a radial offset $\Delta r$ emerges between the opposing capillaries representing the maximum pressure of the gas micropump. While the pressure head p rises with v, $\Delta r$ still tends to vanish towards large $v$ due to the increasing impact of the radially symmetric centrifugal force $\boldsymbol{F}_{v}$ (right graph). And since the optical readout of the radial offset is limited to an accuracy of approx. $\pm 0.4 \mathrm{~mm}$, our principle of measuring $p$ via $\Delta r$ is thus confined to the frequency range below about $30 \mathrm{~Hz}$.

Once the permanent magnet is positioned above the micropump, the air volume in the left arm is pressurized by the pumping pressure $p$ while the air in the right arm remains at the ambient pressure $p_{0}$. This leads to a radial offset $\Delta r$ between the opposing menisci of the plug. The asymmetry stabilizes under the impact of the asymmetric pumping pressure $\Delta p$ and the counteracting centrifugal force $\boldsymbol{F}_{v}$, and the static equilibrium of $\Delta r$ represents a measure for the maximum (no-flow) pumping pressure.

This pressure can be derived from

$$
p=\rho \omega^{2} \Delta r \bar{r}
$$

with the liquid density $\rho$, the angular velocity $\omega=2 \pi v$ and the mean radial position of the water plug $\bar{r}$. The results of these measurements are depicted in Fig. 4 (right). For low frequencies of rotation $v<33 \mathrm{~Hz}$, the pumping pressure increases almost linearly with the frequency $v$ up to about $4.1 \mathrm{kPa}$. Beyond $v=33 \mathrm{~Hz}$, the symmetric centrifugal force $\boldsymbol{F}_{v} \sim v^{2}$ tends to level off $\Delta r$, thus limiting the observable range of pumping pressures $p$ since the optical read of $\Delta r$ from the stroboscopic gray-scale images is limited to approx. $\pm 0.4 \mathrm{~mm}$. Due to the limited resolution of $\Delta r$, the maximum pumping pressure as well as the maximum operational frequency where the phase relation to establish the sequence of plate deflection is destroyed and/or the amplitude of membrane deflection becomes too low cannot be fully explored. At frequencies lower than $10 \mathrm{~Hz}$, the meniscus in the right arm lifts the plug through the air vent.

Fig. 5 visualizes how the pumping pressure can be harnessed for injecting ambient air into a continuous, centrifugally driven liquid stream (ink-colored water).

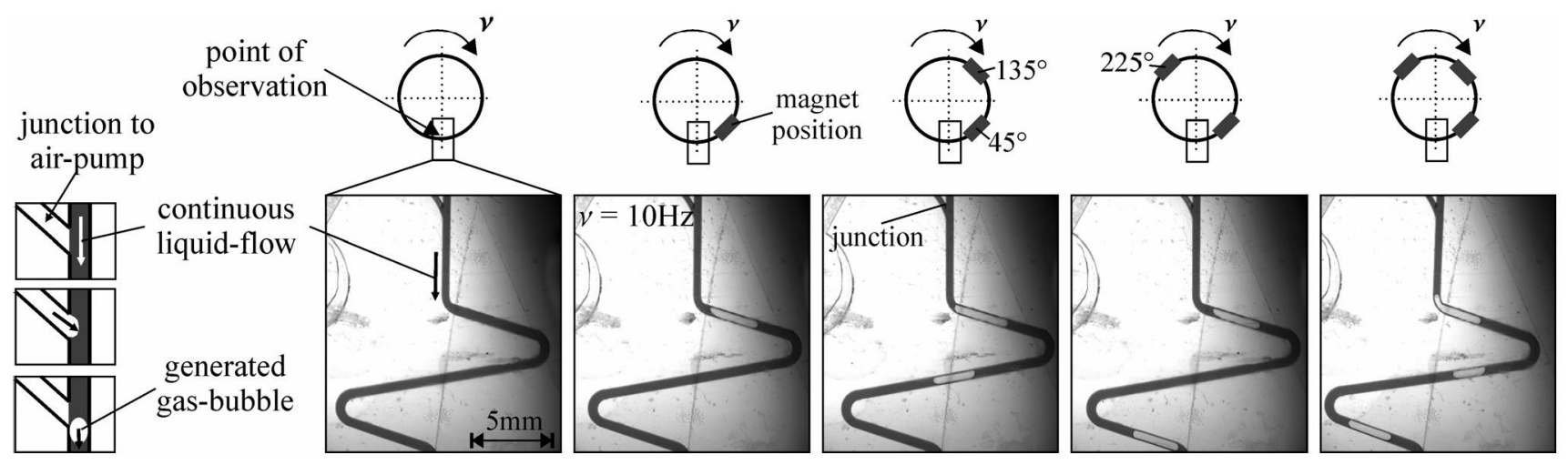

Figure 5: Different modes of gas-liquid flow at a rotational frequency of $v=10 \mathrm{~Hz}$ within a meander shaped microchannel (width: $700 \mu \mathrm{m}$, depth: $200 \mu \mathrm{m}$ ). The introduction of gas bubbles is sketched on the left hand side and the azimuthal positions of the magnets with respect to the camera window are indicated on top of each fotography. The production rate of the gas bubbles depends on the number and the alignment of the permanent magnets along the orbit of the rotating micropump. Without any magnets, no gas-bubbles are generated, thus validating the functional principle to the centrifugo-magnetic pump. 
At a given frequency of rotation $v=10 \mathrm{~Hz}$, the bubble rate changes with the number and the azimuthal position(s) of the permanent magnets which can be placed at $45^{\circ}$, $135^{\circ}$ and $225^{\circ}$ with respect to the point of observation. The number of magnets and the frequency $v$ also set the volume ratio between gas and liquid phase.

\section{SUMMARY AND OUTLOOK}

We presented for the first time a centrifugo-magnetically actuated gas micropump. Pumping is implemented by the phase-shifted displacement of two metal inlays incorporated in the elastic lid of the passive and possibly disposable polymer disk. The new functional principle as well as the fabrication method well comply with the modular approach of centrifugal microfluidic platforms. Future work will be done on the integration of this centrifugo-magnetical gas micropump into a multiphase flow platform. It will add capabilities such as air-to-liquid sampling, e.g. for environmental pathogen detection, and for the controlled admission of pressure pulses on liquid plugs or flows, e.g. for droplet ejection.

\section{ACKNOWLEDGEMENT}

The authors would like to thank Dr. Ralf Förster from the IMTEK Laboratory of process technology who conducted the WEDM machining, as well as Gerhard Jobst for the micromachining of the polymer substrates.

\section{REFERENCES}

[1] M. Madou and G. Kellogg, "LabCD: A Centrifugebased Microfluidic Platform for Diagnostics," in Proceedings of SPIE, 3259 ed 1998, pp. 80-93.

[2] G. Ekstrand, C. Holmquist, A. E. Örlefors, B. Hellman, A. Larsson, and P. Andersson, "Microfluidics in a Rotating CD," in Proceedings of Micro Total Analysis Systems 2000, pp. 311-314.

[3] J. Kim, J. S. Hee, G. Jia, J. V. Zoval, N. A. Da Silva, and M. J. Madou, "Cell lysis on a microfluidic CD (compact disc)," Lab on A Chip, vol. 4, no. 5, pp. 516-522, 2004.

[4] S. Haeberle, T. Brenner, H. P. Schlosser, R. Zengerle, and J. Ducrée, "Centrifugal micromixer," Chemical Engineering \& Technology, vol. 28, no. 5, pp. 613-616, 2005.

[5] L. Riegger, M. Grumann, T. Brefka, J. Steigert, C. P. Steinert, T. Brenner, R. Zengerle, and J. Ducrée, "Bubble-free Priming Of Blind Capillaries For
High-accuracy Centrifugal Hematocrit Measurements," in Proceedings 9th International Conference on Miniaturized Systems for Chemistry and Life Science ( $\mu$ TAS) Boston, USA: 2005, pp. 796-798.

[6] S. Haeberle, R. Zengerle, and J. Ducrée, "Monodisperse Droplet Trains and Segmented Flow for Centrifugal Microfluidics," in Proceedings 9th International Conference on Miniaturized Systems for Chemistry and Life Sciences ( $\mu T A S$ ) Boston, USA: 2005, pp. 635-637.

[7] A. Günther, M. Jhunjhunwala, M. Thalmann, M. A. Schmidt, and K. F. Jensen, "Micromixing of miscible liquids in segmented gas-liquid flow," Langmuir, vol. 21, no. 4, pp. 1547-1555, 2005.

[8] M. Grumann, A. Geipel, L. Riegger, R. Zengerle, and J. Ducrée, "Batch-mode mixing on centrifugal microfluidic platforms," Lab on A Chip, vol. 5, no. 5 , pp. 560-565, 2005.

[9] C. Yamahata, C. Lotto, E. Al-Assaf, and M. A. M. Gijs, "A PMMA Valveless Micropump Using Electromagnetic Actuation," Microfluidics Nanofluidics, vol. 1, no. 3, pp. 197-207, 2005.

[10] T. R. Pan, S. J. McDonald, E. M. Kai, and B. Ziaie, "A magnetically driven PDMS micropump with ball check-valves," Journal of Micromechanics and Microengineering, vol. 15, no. 5, pp. 1021-1026, 2005.

[11] C. Yamahata, F. Lacharme, Y. Burri, and M. A. M. Gijs, "A Ball Valve Micropump in Glass Fabricated by Powder Blasting," Sensors and Actuators $B$ Chemical, vol. 110, pp. 1-7, 2005.

[12] J. Goulpeau, D. Trouchet, A. Ajdari, and P. Tabeling, "Experimental study and modeling of polydimethylsiloxane peristaltic micropumps," Journal of Applied Physics, vol. 98, no. 4, 2005.

[13] M. A. Unger, H. P. Chou, T. Thorsen, A. Scherer, and S. R. Quake, "Monolithic microfabricated valves and pumps by multilayer soft lithography," Science, vol. 288, no. 5463, pp. 113-116, 2000.

[14] M. Grumann, T. Brenner, C. Beer, R. Zengerle, and J. Ducrée, "Visualization of flow patterning in highspeed centrifugal microfluidics," Review of Scientific Instruments, vol. 76, no. 2, 025101, 2005. 